Artículo

\title{
Orientaciones y evolución de la política agraria común (PAC): entre la sostenibilidad y el desarrollo rural
}

\author{
María Elena Serrano Flores ${ }^{1 \S}$ \\ José Manuel del Barrio Aliste ${ }^{2}$ \\ María Luisa Ibáñez Martínez ${ }^{2}$ \\ ${ }^{1}$ Centro Interdisciplinario de Investigaciones y Estudios sobre Medio Ambiente y Desarrollo-Instituto \\ Politécnico Nacional, Ciudad de México. ${ }^{2}$ Facultad de Ciencias Sociales-Universidad de Salamanca, \\ España.(mibanez@usal.es; josema@usal.es). \\ ${ }^{\S}$ Autora para correspondencia: meserrano@ipn.mx.
}

\section{Resumen}

Una de las variables que desde la década de los 80 del pasado siglo han modificado al mundo rural, en su amplia acepción, es sin lugar a dudas el tema de la sostenibilidad y en el abanico de experiencias internacionales resalta la de la Unión Europea que; a través, de la política agraria común (PAC), ha introducido como componente central las medidas agroambientales que han redefinido tanto las orientaciones como las funciones de la agricultura, de los campesinos y de sus territorios, con los consecuentes efectos tanto en la conceptualización como en la práctica del desarrollo rural. Se presenta un análisis histórico del proceso y evolución que ha seguido esta política europea, particularmente su expresión en España. La hipótesis que subyace es que, el cuidado ambiental y la orientación del desarrollo rural de la PAC, se han definido en función del equilibrio de los mercados de los productos agrarios. Esta revisión histórica toma como punto de partida la década de los 90 en que aparece por primera vez la variable agroambiental en la PAC y se sigue su proceso hasta la reforma de 2016, actualmente vigente. El estudio se basa en fuentes bibliográficas primarias: documentos oficiales elaborados por la Unión Europea y entrevistas con informantes clave relacionados con la adecuación y aplicación de los programas de la PAC, particularmente en España. Los resultados comprueban una tendencia en la que el control de precios y el mercado de los productos agropecuarios, particularmente los cereales, ha determinado que la PAC se reoriente hacia la consolidación de las variables agroambientales y que el desarrollo rural, en aras de la multifuncionalidad y diversificación productiva, sea encauzado hacia actividades no agropecuarias.

Palabras clave: desarrollo rural, medidas agroambientales, política agraria común.

Recibido: febrero de 2020

Aceptado: mayo de 2020 


\section{Introducción}

Como se señalaba anteriormente, el mundo rural ha estado sujeto a la aplicación de nuevas orientaciones políticas que han ido modificando paulatina pero sustancialmente su tradicional dinámica económica, social y cultural, así como el uso de sus espacios físicos y recursos naturales. Gran parte de estas modificaciones han sido motivadas tanto por los problemas del mercado de productos agropecuarios como por el deterioro de recursos naturales presente a escala global.

El desarrollo sostenible ha permeado, por tanto, buena parte de la política agrícola donde la sostenibilidad se ha erigido en el eje de una nueva orientación para el sector rural. El calificativo de 'sostenible', vocablo utilizado y generalizado como traducción del inglés 'sustainable development', está interviniendo y definiendo nuevas funciones para la agricultura, los campesinos, sus espacios rurales y obviamente, sus formas de producción y relación con los ecosistemas en los que habitan.

La política agraria común (PAC), es fiel reflejo de este 'progresivo proceso de «ambientalización» de la agricultura' (Gómez et al., 1996). Ambientalización que, de acuerdo con estos autores, podría interpretarse en dos sentidos: la limitación de las prácticas agrícolas perjudiciales al medio ambiente relacionadas con el modelo productivista agroindustrial y el reconocimiento del papel ecológico que históricamente ha desempeñado la agricultura tradicional.

En ese sentido, la PAC ha seguido una evolución que ha ido derivando hacia la consolidación de dos vertientes principales: la ambientalización del campo, que se concreta en las medidas agroambientales y la versión europea del desarrollo rural expresada en los programas por sus siglas en francés de: Liaison entre Actions de Développement de l'Economie Rural (LEADER), cuya propuesta se sintetiza en los conceptos de multifuncionalidad y diversificación de las actividades agrarias y de los espacios rurales, acompañados de nuevas pautas de identificación sociocultural de lo rural, entre los más destacables.

Este nuevo referente en las políticas agrarias genera importantes incertidumbres sobre el futuro que desde instancias ajenas se diseña para el mundo rural, el papel asignado a la agricultura y a los espacios rurales. Por ejemplo, Alario (2000) señala que 'hay dos vertientes que no están suficientemente documentadas: los procesos de cambio económico y social que están teniendo lugar en los diferentes espacios rurales y qué parte de explicación de estos procesos de cambio tienen lo que consideramos como políticas de desarrollo rural'.

\section{Materiales y métodos}

La investigación se realizó a través de consulta bibliográfica y documental, principalmente de autores españoles, así como fuentes primarias (documentos oficiales tanto de las instituciones del gobierno español como del Parlamento Europeo). Se realizaron también entrevistas a académicos y funcionarios de instituciones públicas encargadas de la aplicación y seguimiento de los programas de la PAC y el programa LEADER en España. 
Aunque en este trabajo no se alude a la información proporcionada por los agricultores, sí importa señalar que se realizaron entrevistas y cuestionarios a productores de la localidad rural de Villafáfila (Castilla y León, España), beneficiarios de los programas agroambientales, que permitieron afianzar la información sobre la orientación general de los programas de la PAC, particularmente las medidas agroambientales y los programas de desarrollo rural.

\section{Resultados y discusión}

\section{Planteamientos iniciales y evolución hacia la ‘ambientalización’ y el desarrollo rural}

En el convulso escenario económico, político y social de los años 80 del pasado siglo, la entonces Comunidad Económica Europea inició un importante proceso de reformas a la PAC, inducidas de alguna manera por la explosión de la crisis agrícola mundial y la disputa internacional de mercados y precios. A lo largo de esa década y principios de la siguiente, la PAC irá redefiniendo el papel de la agricultura, de los espacios rurales y de los campesinos en el contexto del desarrollo económico europeo, perfilando desde entonces una orientación hacia el control de mercados y precios obviamente, pero también hacia la agroambientalización, diversificación y multifuncionalidad de los espacios rurales.

En la historia de la evolución de la política ambiental europea, destacan los siguientes hechos: a) en 1990 se crea la Agencia Europea de Medio Ambiente (AEMA), con sede en Copenhague; b) en 1992 se crea el Programa Natura 2000, financiado a través del Programa LIFE, instrumento político para la definición de la red de zonas naturales protegidas, la conservación de la naturaleza y la protección del medio ambiente; c) el Tratado de Maastrich de 1993, otorga carácter oficial al medio ambiente en los programas de la Unión Europea; d) el Tratado de Amsterdam, 1999, establece la obligación de integrar la protección medioambiental en todas las políticas sectoriales de la Unión Europea con objeto de promover el desarrollo sostenible; e) en el año 2000, se presenta el Libro Blanco sobre Responsabilidad Ambiental, que establece el principio "quien contamina paga'; y f) El Tratado de Lisboa en 2009, plantea el compromiso de luchar contra el cambio climático.

En el año 2013, el Consejo y el Parlamento Europeo adoptaron el séptimo programa de acción en materia de medio ambiente con vigencia hasta el año 2020. El documento titulado 'vivir bien, respetando los límites de nuestro planeta', establece nueve objetivos prioritarios, entre los que destacan: la protección de la naturaleza; una mayor resistencia ecológica; crecimiento sostenible y eficiente en el uso de los recursos e hipocarbónico; la lucha contra las amenazas para la salud relacionadas con el medio ambiente (Diario Oficial de la Unión Europea, 2013). La variable del cuidado medioambiental en las políticas de la Unión Europea no se ha limitado al ámbito de lo biológico o ecológico per se, sino que paulatinamente se han integrado en la PAC, como se muestra en el Cuadro 1.

Cuadro 1. La variable ambiental en la política agraria común. Proceso histórico de las modificaciones más relevantes 1985-2017.

\begin{tabular}{cl}
\hline Año & \multicolumn{1}{c}{ Reforma } \\
\hline 1985 & $\begin{array}{l}\text { Primera reforma de la PAC. Se presenta el Libro Verde que plantea la orientación de la } \\
\text { agricultura europea hacia el año 2000. }\end{array}$ \\
\hline
\end{tabular}




\begin{tabular}{cl}
\hline Año & \multicolumn{1}{c}{ Reforma } \\
\hline 1988 & $\begin{array}{l}\text { La CEE presenta el informe 'el futuro del mundo rural' en el que por primera vez se habla } \\
\text { de objetivos como fijar a la población rural, cuidado del paisaje y del medio ambiente. }\end{array}$ \\
& $\begin{array}{l}\text { Este documento marca también el inicio de la iniciativa LEADER. } \\
\text { Sobre la base del Libro Verde, se aprueba el llamado 'paquete delors' que establece } \\
\text { mecanismos para desalentar la producción agraria, como la reducción de los precios de } \\
\text { garantía en 6\%, fijación de cuotas de producción, programa de retiro de tierras de la } \\
\text { producción, extensificación y reconversión de cultivos excedentarios por otros no } \\
\text { excedentarios, reforma de los fondos estructurales FEDER, FSE y FEOGA-O, dividiendo } \\
\text { el territorio de la CEE en 5 tipos de zonas. }\end{array}$
\end{tabular}

1991 Se elabora un nuevo documento 'evolución y futuro de la PAC', conocido también como Informe MacSharry que reconoce el fracaso de la primera reforma y la continuidad en el crecimiento del gasto agrícola, condensa la nueva orientación de la PAC, esbozada desde 1988: la plurifuncionalidad de la agricultura (no más productores de alimentos sino conservadores y protectores del medio ambiente), mantenimiento de la población agraria en el medio rural; diversificación de las actividades económicas para complementar las rentas de los agricultores. Entra en operación el programa de desarrollo rural LEADER I (1991-1993), en el que se plantea una orientación hacia aspectos como: turismo, artesanía, y conservación medio ambiental.

1992 Reforma Mac Sharry. Introducción de los programas agroambientales como medidas de acompañamiento de la PAC (Diario Oficial de las Comunidades Europeas, 1992). Esta reforma tuvo su aplicación durante tres campañas agrícolas: 1993-1994, 1994-1995 y 1995-1996. Se reitera y confirma la orientación que para el futuro del mundo rural se planteaba ya en el documento presentado en 1988.

1994 Puesta en operación de la segunda versión del programa LEADER II.

1995 Revisión de la PAC. Se plantean, entre otras medidas: mejorar la competitividad reduciendo los precios institucionales; fomento de prácticas respetuosas con el medio ambiente y la diversificación de las actividades en el medio rural.

1999 Nueva revisión de la PAC. Siguiendo la orientación de la reforma de 1992, se refuerza la intención de mejorar la competitividad mediante la reducción de precios institucionales y se fortalece la vía del desarrollo rural en detrimento de la de precios y mercados. Se establece el desarrollo rural como segundo pilar de la PAC.

2000 Puesta en operación de la tercera versión del programa de desarrollo rural, denominado LEADER+

2003 Conocida como «revisión intermedia» o «perspectiva política a largo plazo para una agricultura sostenible», se aprueba una nueva reforma de la PAC que desvincula de la producción las ayudas directas al ingreso de los agricultores. La política de precios y mercados cede su lugar al desarrollo rural. Desacoplamiento, modulación y mayor importancia al desarrollo rural son las grandes medidas adoptadas y de aplicación a partir del año 2007, vigentes hasta 2013. Destaca la creación del Fondo Europeo para la Agricultura y el Desarrollo Rural (FEADER), la modificación del FEOGA, que pasa a denominarse FEGA dirigido a financiar el primer pilar de la PAC: la política de mercados García-Delgado y García-Grande (2005).

2009 Conocida como 'el chequeo de la PAC', se aprueba el 20 de noviembre de 2008 y plantea reforzar la disociación total de las ayudas mediante la eliminación progresiva de los últimos pagos asociados a la producción, integrándolos en el régimen de pago único por explotación y reorientar parcialmente fondos del primer pilar a favor del desarrollo rural. 


\begin{tabular}{cl}
\hline Año & \multicolumn{1}{c}{ Reforma } \\
\hline 2013 & Última fase del proceso de adaptación de la PAC. Entre las modificaciones más relevantes \\
& se encuentran: los pagos únicos a las explotaciones se sustituyen por un sistema de pagos \\
por niveles o estratos con siete componentes: 1) un 'pago básico'; 2) un 'pago verde' para & \\
bienes públicos medioambientales (componente ecológico); 3) un pago complementario \\
a los agricultores jóvenes; 4) un pago redistributivo para reforzar la ayuda a las primeras \\
hectáreas de una explotación; 5) una ayuda adicional a los ingresos en las zonas \\
condicionadas por limitaciones naturales; 6) ayudas vinculadas a la producción; y 7) \\
régimen simplificado en favor de los pequeños agricultores. Se consolida el desarrollo \\
rural como segundo pilar de la PAC. \\
En el mes de septiembre, la Comisión Europea organiza la Conferencia de Cork 2.0, que \\
reabre el debate en torno a la política de desarrollo rural que se aplicará a partir del año \\
2020. \\
La reunión da como resultado que en noviembre de este año se presente una nueva \\
comunicación sobre el futuro de la alimentación y de la agricultura que hace énfasis \\
principalmente en el desarrollo sostenible y la preservación de los recursos naturales.
\end{tabular}

FEDER = fondo europeo para el desarrollo rural; FSE= Fondo social europeo; FEOA-O= fondo europeo de orientación y garantía agrícola. Elaboración con base en: Garrido (2000); García-Delgado; García-Grande (2005); Moyano (2005); Diario Oficial de la Unión Europea (1991, 1992, 2013).

Esta cronología refleja cómo, paulatinamente, la visión productivista de la agricultura va virando hacia la componente medioambiental. Como señalan Sumpsi et al. (1997), en el Libro Verde de 1985 'que representa la posición de la PAC hacia el año 2000, se planteaba por primera vez la necesidad de que se incluyeran las cuestiones ambientales y se reconocía como una de las funciones de la agricultura la conservación del entorno natural'. Otro elemento destacable en esta historia es la aparición del programa LEADER orientado hacia la promoción del desarrollo rural que, desde la perspectiva europea, tiene un sentido diferente del desarrollo agrícola-productivo.

\section{La orientación medioambiental de la política agraria común}

Con poco éxito en sus resultados, el aspecto medioambiental de la agricultura es introducido en la PAC; a través, de la política agraria de estructuras de la década de los 70, por ejemplo, la directiva 159/72 sobre el cese de la actividad agrícola y la 161/72 sobre formación profesional de los agricultores (Garrido, 2000), pero es hasta la década de los 80 que el aspecto medioambiental va a figurar más claramente en los planteamientos y objetivos de la PAC.

El Libro Verde de 1985 condensa la expresión por las preocupaciones medioambientales y el reglamento CEE 797/85 es el instrumento donde se plasma la disposición legal de tal inclusión Garrido (2000) otros reglamentos diseñados para el cuidado ambiental son, por ejemplo, el CEE $787 / 85$ que habla sobre la mejora de la eficacia de las estructuras agrarias y el artículo 21 de este reglamento que establece por primera vez un sistema de ayudas para la repoblación forestal de superficies agrícolas y la conservación de masas forestales en las explotaciones agrarias Sumpsi et al. (1997). Otro instrumento legal establecido en la PAC es el reglamento CEE 2328/91 (Diario Oficial de la Comunidad Europea, 1991), donde se deja claro como uno de sus objetivos y por tanto objetivo de la política estructural comunitaria, la contribución a la protección del medio ambiente y al mantenimiento del espacio rural, incluida la conservación duradera de los recursos naturales de la agricultura. 
Otras de las medidas importantes hacia la consolidación de la orientación ambiental de la PAC es la referente a los fondos estructurales FEDER, FSE Y FEOGA-O que se reforman en 1988 con objeto de introducir más abiertamente la cuestión medioambiental en las políticas agrarias comunes. Esta reforma otorga al FEOGA-O la función de 'contribuir al entramado social de las zonas rurales, a la protección del medio ambiente, a la conservación del espacio rural (incluida la de los recursos naturales de la agricultura) y a compensar los efectos que tienen los obstáculos naturales para la agricultura' (Garrido, 2000). En el año 2003 se crea el Fondo Europeo para la agricultura y el desarrollo rural (FEADER) y se modifica el FEOGA que se transforma en FEGA dirigido a financiar la política de mercados, primer pilar de la PAC.

Cabe enfatizar, sin embargo, que es en la reforma de 1992 donde se define su orientación ambiental a través de las medidas agroambientales consideradas como medidas de acompañamiento. El Reglamento 2078/92 denominado 'métodos de producción agraria compatibles con las exigencias de protección del medio ambiente y la conservación del espacio natural', establecía desde entonces el objetivo de que la agricultura adquiriera prácticas más respetuosas con el medio ambiente.

El funcionamiento administrativo para apoyar a los agricultores que aceptaran acogerse a ese programa agroambiental resulta también singular ya que 'se establece un sistema de contratación individualizada entre el agricultor y los poderes públicos: el agricultor acepta unos compromisos respecto al tipo de prácticas a realizar en su explotación a cambio de recibir una ayuda económica procedente de fondos públicos para compensar la menor rentabilidad que le pudiera ocasionar la opción por una agricultura sostenible' (Garrido, 2000).

Estas ayudas, se destinarían únicamente a compensar las medidas que tengan efectos positivos para el medio ambiente en los siguientes aspectos: reducción en el uso de fertilizantes y productos fitosanitarios, mantener las reducciones ya iniciadas o introducir y mantener métodos de agricultura biológica, extensificar las producciones vegetales, incluidas las forrajeras o transformar tierras de cultivos herbáceos en pastizales extensivos, reducir la cabaña bovina y ovina, criar animales de razas locales en peligro de desaparición, mantener en buen estado las tierras de labor o forestales retiradas de la producción, retirar de la producción tierras de labor durante al menos 20 años para constituir reservas de biotopos, parques naturales o para proteger las aguas, incluir medidas destinadas a mejorar la formación de los agricultores sobre prácticas de producción compatibles con el medio ambiente.

La producción de alimentos y la reproducción social y cultural de la población rural, prácticamente se subordina a la conservación de los recursos naturales. La pérdida económica que significa abandonar o subordinar la actividad agrícola por la conservación del ecosistema es compensada por la Unión Europea; a través, de una serie de incentivos, subsidios o ayudas económicas, como antes se señaló. Para ejemplificar el tipo de programas y monto de los subsidios, en el Cuadro 2 se presenta el caso de la localidad de Villafáfila (Castilla y León, España), considerado paradigmático respecto a la aplicación de las medidas agroambientales. Paniagua (1999), señala que para 1999, España es el país con mayor número de estos programas (66) de toda la Unión Europea. 
Cuadro 2. Programas agroambientales y compromisos (Villafáfila, Castilla y León, Campañas 2007-2014).

\begin{tabular}{|c|c|c|}
\hline Programa & Compromisos obligatorios & $\begin{array}{l}\text { Ayuda anual } \\
\text { (euros) }\end{array}$ \\
\hline \multirow[t]{2}{*}{$\begin{array}{l}\text { Agroecosistemas } \\
\text { extensivos de } \\
\text { secano }\end{array}$} & $\begin{array}{l}\text { Llevar y mantener actualizado el cuaderno de la explotación, } \\
\text { no utilizar semillas tratadas con productos fitosanitarios } \\
\text { salvo si pertenecen a la categoría 'baja peligrosidad' para el } \\
\text { hombre y la fauna terrestre y acuícola, mantener un } 3 \% \text { de la } \\
\text { superficie básica en forma de linderos o islas de vegetación } \\
\text { espontánea. Esta superficie permanecerá fija sin cultivar } \\
\text { durante todo el periodo de duración del compromiso; } \\
\text { incrementar las dosis habituales de siembra en el cultivo de } \\
\text { cereales, estableciéndose ciertas dosis para cumplir este } \\
\text { compromiso. }\end{array}$ & $65 € \mathrm{ha}^{-1}$ \\
\hline & $\begin{array}{l}\text { En parcelas que vayan a dedicarse a la retirada o barbecho } \\
\text { tradicional, en la campaña siguiente deberá mantenerse el } \\
\text { rastrojo hasta el } 01 \text { de febrero siguiente; la recolección de } \\
\text { cereales no se podrá efectuar hasta después de las fechas } \\
\text { fijadas para cada comarca, dedicar } 15 \% \text { de la superficie } \\
\text { acogida al cultivo de leguminosas y proteaginosas, para } \\
\text { grano o forraje, dedicar } 10 \% \text { de la superficie anual acogida } \\
\text { al cultivo de cereales de ciclo largo, cumplir con los } \\
\text { requisitos legales y las buenas condiciones agrarias y } \\
\text { medioambientales que les sean de aplicación, cumplir con } \\
\text { los requisitos mínimos de fertilización, fitosanitarios, etc. } \\
\text { Como compromiso voluntario se establece destinar el } \\
\text { barbecho tradicional a barbecho semillado con leguminosas } \\
\text { para la alimentación de las aves, el enterrado en verde o el } \\
\text { pastoreo, no pudiendo realizar otro tipo de aprovechamiento } \\
\text { en estas superficies. }\end{array}$ & $\begin{array}{c}50 € \mathrm{ha}^{-1} \\
\text { adicionales al } \\
\text { apoyo } \\
\text { anterior }\end{array}$ \\
\hline $\begin{array}{l}\text { Cultivo de } \\
\text { girasol en } \\
\text { secano en zonas } \\
\text { Red Natura } \\
2000 .\end{array}$ & $\begin{array}{l}\text { Compromisos durante cinco campañas agrícolas: cultivar la } \\
\text { superficie de girasol en parcelas ubicadas en municipios } \\
\text { incluidos en la Red Natura 2000, llevar un cuaderno de } \\
\text { explotación; mantener las linderas existentes, enterrar el } \\
\text { rastrojo del cereal precedente al girasol antes del } 01 \text { de } \\
\text { febrero siguiente a la cosecha de cereal, realizar la siembra } \\
\text { de girasol en dosis no inferiores a } 325 \mathrm{~kg} \mathrm{ha}^{-1} \text {, no utilizar } \\
\text { herbicidas en el cultivo del girasol, no emplear abonos de } \\
\text { síntesis química, mantener el rastrojo del cultivo de girasol } \\
\text { sin pastorear; triturar y enterrar el cañote del girasol, cumplir } \\
\text { con las buenas condiciones agrarias y medioambientales que } \\
\text { les sean de aplicación, cumplir con los requisitos mínimos de } \\
\text { fertilización, fitosanitarios y otros requisitos obligatorios. }\end{array}$ & $60 € \mathrm{ha}^{-1}$ \\
\hline
\end{tabular}




\begin{tabular}{|c|c|c|}
\hline Programa & Compromisos obligatorios & $\begin{array}{c}\text { Ayuda anual } \\
\text { (euros) }\end{array}$ \\
\hline $\begin{array}{l}\text { Cultivo del } \\
\text { ecotipo de } \\
\text { alfalfa de secano } \\
\text { Tierra de } \\
\text { Campos }\end{array}$ & $\begin{array}{l}\text { Destinar las parcelas acogidas a la ayuda al cultivo del } \\
\text { ecotipo de alfalfa de secano Tierra de Campos y ubicar } \\
\text { dichas parcelas en municipios de la Reserva Natural de las } \\
\text { Lagunas de Villafáfila y en las zonas especiales de } \\
\text { protección ambiental (ZEPA) de la comarca de Tierra de } \\
\text { Campos, mantener el cultivo en adecuado estado de } \\
\text { conservación y con una edad máxima de cinco años; no se } \\
\text { podrán realizar más de dos cortes al año y no se podrá segar } \\
\text { desde el } 15 \text { de mayo hasta el } 1 \text { de julio, prohibición de segar } \\
\text { desde la puesta de sol hasta el amanecer; dejar sin cosechar } \\
\text { al menos } 5 \% \text { en forma de linderas de anchura mínima } 3 \text { m.; } \\
\text { la parcela de cultivo de alfalfa debe cumplir con las } \\
\text { condiciones de idoneidad ambiental: distancia a núcleos } \\
\text { urbanos superior a } 400 \text { m, distancia a vía de comunicación } \\
\text { carretera superior a } 200 \text { m, e inexistencia de tendidos } \\
\text { eléctricos, cumplir con los requisitos legales y la buena } \\
\text { condiciones agraria y medioambientales que se sean de } \\
\text { aplicación; cumplir con los requisitos mínimos de } \\
\text { fertilización, fitosanitarios y otros requisitos obligatorios }\end{array}$ & $300 € \mathrm{ha}^{-1}$ \\
\hline
\end{tabular}

Para el cálculo de la ayuda anual se establecen 45 hectáreas como unidad mínima de cultivo agroambiental (UMCA) para la superficie total acogida. Hasta el doble de la UMCA, se percibe $100 \%$ de la ayuda, por la superficie superior al doble de la UMCA y hasta el cuádruple, 60\% del importe, por la superficie superior al cuádruple de la UMCA, 30\% del importe. La diferencia entre los apoyos económicos otorgados radica en criterios de orden ambiental, por ejemplo, la alfalfa tuvo un apoyo económico mayor pues su cultivo se considera importante para la diversidad biológica y por ser parte del hábitat de la avutarda, especie en peligro de extinción. Elaboración con base en: orden AYG/1111/2010, de 23 de julio, período de programación 2007-2013 (Boletín Oficial de Castilla y León, No 149).

\section{El desarrollo rural como segundo pilar de la política agraria común}

El surgimiento del desarrollo rural como segundo pilar de la PAC corre paralelo al proceso de agroambientalización -medida de acompañamiento de la PAC-. Incluso, afirmarían Colino-Sueiras y Martínez-Paz (2005), sus antecedentes se encontrarían desde el Tratado de Roma y subsecuentes reformas y conferencias en las que se ha ido transfiriendo el énfasis de lo sectorial hacia lo territorial.

Resulta importante notar que en el documento 'el futuro del mundo rural', se identifican problemas como el detrimento del medio ambiente, el declive rural -entendido como la emigración de la población joven hacia las ciudades con el proceso de envejecimiento consecuente- y en tercer lugar, el problema de las zonas marginadas especialmente la agricultura de montaña, amenazadas por el declive agrario y la despoblación. 
Este diagnóstico determina que la orientación del desarrollo rural se concretice en un sentido «no agrario» contenido en los programas de las Iniciativas Comunitarias como: Urban, Interreg y LEADER. Iniciativas concebidas, señalan Molinero y Alario (1994) 'como la forma de incentivar el desarrollo rural a escala comarcal a través de iniciativas endógenas destinadas a la potenciación de los aspectos que hoy se consideran fundamentales: turismo (45\% del presupuesto), artesanía (21\%), actividades agrarias alternativas a las tradicionales, conservación medioambiental, entre otras, teniendo prioridad las que aportan soluciones innovadoras, diversifican la base económica comarcal y dan alternativas a las actividades agrarias clásicas'. Aunque existe un reconocimiento casi generalizado sobre el éxito de este programa, Sancho (2002) indica que habría que tomar en cuenta la cuestión relativa a la participación de la sociedad rural más que enfocarse en los beneficios económicos, 'pues sería en aquélla donde la iniciativa del LEADER dejaría más que desear'.

De esta manera, el LEADER que puede considerarse como la primera puesta en práctica de una política rural de carácter territorial, integrada y participativa, está destinada al sostenimiento y desarrollo de iniciativas locales de desarrollo rural cuyos rasgos específicos se sintetizan en siete puntos: 1) el enfoque territorial, lo que supone basarse en los recursos particulares de cada comarca, para así responder mejor a las necesidades locales; 2) el enfoque ascendente en la búsqueda de soluciones y en la toma de decisiones, con el propósito de conceder el mayor protagonismo posible a los agentes locales; 3) el grupo de acción local como forma de cooperación horizontal, agrupando a los agentes e instituciones locales y comarcales representativos; 4) el carácter innovador de las acciones promovidas por los beneficiarios respecto de otras intervenciones en la zona rural afectada; 5) el enfoque integral e intersectorial, al contemplarse conjuntamente las potencialidades de los diferentes sectores de la economía, la sociedad y los recursos locales; 6) la descentralización de la gestión y la financiación, mediante los grupos de acción local; y 7) la organización en red y la cooperación transnacional, no sólo para el intercambio de conocimientos, experiencias y resultados, sino también a través de la asociación en proyectos comunes con grupos de otros países (Colino-Sueiras y Martínez-Paz, 2005).

La Conferencia Europea sobre desarrollo rural celebrada en la ciudad irlandesa de Cork el 9 de noviembre de 1996, bajo el título 'la Europa rural: perspectivas de futuro', aprueba la Declaración de Cork, donde se establece un decálogo cuyo primer precepto asienta la preferencia rural como prioridad en las políticas de la Unión Europea, los nueve restantes reiteran elementos de anteriores declaraciones de principios que, en gran medida, se venían aplicando con los programas LEADER.

El desarrollo rural comprende entonces, actuaciones horizontales con objetivos diversos entre los que cabe mencionar: lograr un sector agrario viable, evitar el despoblamiento rural y preservar el patrimonio natural. En esos años se consideraba que para alcanzar estos objetivos se debían conceder subvenciones para el desarrollo de actividades diversas relacionadas con el medio ambiente, el turismo rural, la agroindustria, la silvicultura o la instalación de jóvenes agricultores, por citar sólo algunos ejemplos.

Las prioridades que se otorgan al desarrollo rural son, entre otras: a) fomentar la competitividad de la agricultura; b) garantizar la gestión sostenible de los recursos naturales y la acción por el clima; y c) lograr un desarrollo territorial equilibrado de las economías y comunidades rurales que incluya la creación y la conservación del empleo (Parlamento Europeo, 2018b). Consecuentemente, la Comisión Europea establece como medidas a ser apoyadas por el Fondo Europeo Agrícola de 
Desarrollo Rural: asesoramiento, inversiones, explotaciones agrícolas, medidas forestales, medidas agroambientales, agricultura ecológica, servicios de medio ambiente, zonas con limitaciones naturales, cooperación, programa LEADER (MAGRAMA, 2016).

\section{Conclusiones}

La integración de España a la Unión Europea implica, como señala Regidor (2002), 'un proceso incesante de ajustes, en los que la agricultura comercial ha sido la que ha acaparado la mayor parte de los recursos económicos y también la que mejor se ha podido adaptar o integrar a la economía europea'. En contraparte, la agricultura que él llama territorial ha sido sometida a un proceso continuo de reducción y supresión a pesar de su importancia espacial, social y económica. En este sentido y como se ha mostrado, los ejes sobre los que se ha hecho descansar a la PAC han tenido como objetivo el equilibrio económico interno y la competencia en el mercado externo, de ahí que el primer pilar de la PAC haya sido la política de precios y mercados que ha sido la más importante en cuanto a recursos proporcionados y actuaciones realizadas.

En ese contexto, la inclusión del cuidado medioambiental y el desarrollo rural, definido como el segundo pilar de la PAC, en su versión no agraria, permite mantener bajo control la producción y los precios agrarios. Habría que señalar, por otro lado, que la información presentada en este artículo no permite concluir si la PAC en sus diferentes componentes políticos y programáticos está generando procesos positivos en materia de conservación de los recursos naturales. Respecto al desarrollo rural bajo la concepción del programa LEADER, al menos en la zona de Castilla y León donde se realizó el presente estudio, no parece haber conseguido sus objetivos.

\section{Agradecimientos}

Este trabajo es parte de una investigación realizada con el apoyo del Consejo Nacional de Ciencia y Tecnología (CONACYT), que financió la estancia sabática en la Universidad de Salamanca, España (octubre 2018-julio 2019).

\section{Literatura citada}

Alario, M. 2000. Las políticas de planificación y de desarrollo de los espacios rurales. In: García Pascual, F. El mundo rural en la era de la globalización: incertidumbres y potencialidades. MAPA, Madrid. 232-294 pp.

BOCYL. 2010. Boletín Oficial de Castilla y León. Número 149, 02 de agosto de Junta de Castilla y León, España.

Colino-Sueiras, J. y Martínez-Paz, J. M. 2005. El desarrollo rural. Segundo pilar de la PAC. In: García-Delgado, J. L. y García-Grande, J. 2005. Política agraria común: balance y perspectivas. Caja de ahorros y pensiones de Barcelona, Colección Estudios Económicos Núm. 34. Barcelona, España. 70-99 pp.

Diario Oficial de la Unión Europea. 2013. 28 de diciembre, decisión No. 1386/2013/UE del Parlamento Europeo y del Consejo, relativa al programa general de acción de la unión en materia de medio ambiente hasta 2020. https://eur-lex.europa.eu/legal-content. 30 p. 
Diario Oficial de las Comunidades Europeas. 1991. 06 de agosto, reglamento CEE No. 2328/91 del Consejo, relativo a la mejora de la eficacia de las estructuras agrarias. Consejo Europeo, Bruselas. https://eur-lex.europa.eu/legal-content. $21 \mathrm{p}$.

Diario Oficial de las Comunidades Europeas. 1992. 30 de junio, reglamento CEE No. 2089/92 sobre métodos de producción agraria compatibles con las exigencias de la protección del medio ambiente y la conservación del espacio natural. Consejo Europeo, Bruselas. https://eur-lex.europa.eu/legal-content. 6 p.

García-Delgado, J. L. y García-Grande, J. 2005. Política agraria común: balance y perspectivas. Caja de ahorros y pensiones de Barcelona. Colección Estudios Económicos Núm. 34. Barcelona, España. 59-62 pp.

Garrido, F. F. 2000. La cuestión ambiental en la agricultura: actores sociales y política agroambiental en España. Fundación Unicaja. 90-18 pp.

Gómez-Benito, C.; Noya, J. y Paniagua, A. 1996. Agricultura y naturaleza. Una aproximación a las imágenes y actitudes de la población respecto a las relaciones entre agricultura, medio rural y naturaleza. Política y Sociedad No. 23. Madrid, España. 99-110 pp.

MAGRAMA. 2016. Ministerio de Agricultura, Alimentación y Medio Ambiente. Marco general para la aplicación de la política de desarrollo rural 2014-2020 en España. www.redruralnacional.es.

Molinero, F. y Alario, M. 1994. La dimensión geográfica del desarrollo rural. Una perspectiva histórica. Ministerio de Agricultura, Alimentación y Medio Ambiente, España. Revista de Estudios Agro-Sociales. 169(3):53-87.

Moyano-Estrada, E. 2005. Nuevas orientaciones de la política europea de desarrollo rural. Instituto de Estudios Sociales Avanzados de Andalucía. Consejo Superior de Investigaciones Científicas. Junta de Andalucía, documentos de trabajo. 0514. 22 p.

Paniagua, M. A. 1999. Cambio rural y política agroambiental. El caso del Programa de Estepas Cerealistas de Castilla y León. Anales de Geografía de la Universidad Complutense, Madrid, España. 170-178. pp.

Parlamento Europeo. 2018 (a). Los instrumentos de la PAC y sus reformas. Fichas técnicas sobre la Unión Europea. Consejo Europeo. www.europarl.eu/factsheets/es.

Parlamento Europeo. 2018 (b). El segundo pilar de la PAC: la política del desarrollo rural. Fichas Técnicas sobre la Unión Europea. Consejo Europeo. www.europarl.eu/factsheets/es.

Regidor, J. G. 2002. La agricultura española en la Unión Europea: entre la integración y la reconversión. In: Gómez-Benito, C. y González, J. J. Agricultura y Sociedad en el cambio de siglo. MacGraw Hill, Madrid. 233-237 pp.

Sancho, R. 2002. Las políticas de desarrollo rural. In: Gómez-Benito, C. y González, J. J. Agricultura y Sociedad en el Cambio de Siglo. McGraw Hill-UNED, Madrid. 293 p.

Sumpsi, J. M.; Garrido, A. e Iglesias, E. 1997. La política agroambiental de la UE: un análisis desde la perspectiva económica. In: Ministerio de Agricultura, Pesca y Alimentación, España. Revista Española de Economía Agraria. 179(1):227-265. 